\title{
The Ring Never Relieves
}

Christian Liebchen (TH Wildau), Steffen Dutsch (TU Dresden), Shiguang Jin (TU Dresden), Norio Tomii (Nihon University), Yihui Wang (Beijing Jiaotong University)

\section{Table of Contents}

1 Introduction ...................................................................................... 2

2 Characteristics of Circle Lines Relevant to Delay Management ........ 5

3 Circle Lines in Practice ............................................................. 8

4 Response Rules for Circle Lines .................................................... 13

5 Expert Interviews on Response Rules for Circle Lines ................... 19

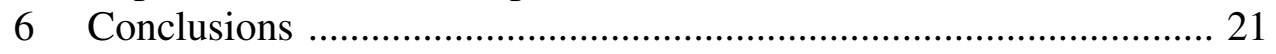

\section{PREPRINT}

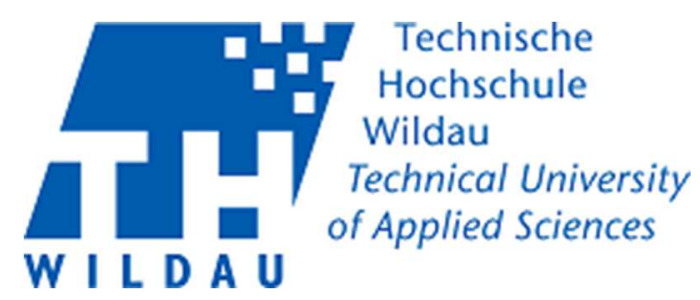

Accepted for publication in the proceedings of the „9th International Conference on Railway Operations, Modelling and Analysis (aka ICROMA 2021)“

Beijing Jiaotong University, Beijing, China

Licence: CC-BY-NC-ND 


\title{
The Ring Never Relieves
}

\author{
Christian Liebchen ${ }^{\mathrm{a}, 1}$, Steffen Dutsch ${ }^{\mathrm{b}}$, Shiguang Jin ${ }^{\mathrm{b}}$, Norio Tomii ${ }^{\mathrm{c}}$, \\ Yihui Wang ${ }^{\mathrm{d}}$ \\ a Technische Hochschule Wildau, Engineering and Natural Sciences, \\ Hochschulring 1, 15745 Wildau, Germany \\ ${ }^{1}$ E-mail: liebchen@th-wildau.de, Phone: +49 (0) 3375508755 \\ b Technische Universität Dresden, Fakultät Verkehrswissenschaften "Friedrich List", \\ Institut für Bahnsysteme und Öffentlichen Verkehr \\ Hettnerstraße 1, 01062 Dresden, Germany \\ ${ }^{\mathrm{c}}$ Nihon University, Center for Railway Research, \\ 1-2-1 Izumicho, Narashino-shi, Chiba 27-8575, Japan \\ d Beijing Jiaotong University, \\ State Key Laboratory of Rail Traffic Control and Safety, \\ No.3 Shuangyuancun, Haidian District, Beijing 100044, China
}

\begin{abstract}
Regarding the disposition of metro lines in order to recover from delays, in the literature there can be found two branches of contributions: On the one hand, there are descriptions of response rules such as expressing (also referred to as skip stop), holding and short-turning together with studies in what situation to apply which of them. On the other hand, there are fully-automated optimization models which make use of any possible driving and routing options, according to some specified objective function.

To the best of our knowledge, we are not aware of any study that puts its focus specifically on ring lines (also known as circle or loop lines). Yet, in the absence in particular of more or less "natural" time buffers in turnaround activities in the endpoints, the operation of a circle line is particularly challenging.

In this spirit, we are collecting response rules that are applicable especially for circle lines. We sketch their impacts on the passengers' travel experience as well as on the resource schedules for the rolling stock and staff (train drivers). For selected response rules, we provide illustrative drawings.

Moreover, we present the answers that experts of eleven metro networks that are operating circle lines were pointing to us during interviews. It turns out that despite the limited possibilities along circle lines there is a broad repertoire of response action that is taken on a regular basis - but also that some standard general response rule (expressing) is almost never applied in practice.
\end{abstract}

\section{Keywords}

Metro Networks, Circle, Loop \& Ring Lines, Delay Management, Response Rules, Expert Interviews

\section{Introduction}

In most agglomerations, metro systems are key to comply with the mobility needs of the citizens. Imagining that all of today's passengers would go by car is commonly considered to be highly negative, both for the traffic flow, and in particular with respect to the environmental impacts. Especially during peak hours, metro lines are the arteries of a city's transportation system. Many metro systems in the world show several millions of 
passengers - each day.

In this contribution, we are putting the focus on lines with a special topology: circle lines. For marketing reasons, in some cities, alternatively, these could also be called ring lines, or loop lines. We are only considering such lines, whose trains are operating continuously for several complete rounds in a row, i.e., not any operating scheme in form of a "lasso", e.g. A-B-C-D-B-A. True circle metro lines have been established in not many more than a dozen cities worldwide. There, they are commonly considered as an attractive service. This is reflected by high demand, which justifies high frequencies. Out of the 12 circle lines that we have been able to collect expert information from, there is only one whose peak hour interval was larger than 5 minutes (5.5 minutes on Line 5 in Zhengzhou, China). In particular, circle lines help to reduce the traffic load from the city center, because they constitute a kind of bypass.

But in the case of disruptions, such true circle lines do not have any natural buffer times during turnaround activities in their terminus stations. This feature makes delay management highly challenging. In the case when failures or even accidents occur, these cause light or serious disruptions on the normal operation of trains and bring inconvenience for the passengers. The shorter the scheduled interval, the larger the relative worsening within only a short time after the occurrence of the actual disruption. At least to some extent, timetables that have been designed under the principle of robustness could absorb delays, but not in any case, of course.

In the past, there have been carried out several studies on delay management in particular for metro services. While we are going to concentrate on managing relatively small disruptions for the specific case of circle lines, in the literature these two features are merely covered separately. Huang et al. (2020) studied the operations of a circle line - but they are considering the severe disruption of a partial track blockage, where two recovery strategies (i.e., the alternating operations on the remaining track, and the short-turning operations) are compared.

For the case of non-circle metro lines - as they pose the vast majority among all metro lines - many different response rules had been discussed in the literature. For instance, Shen and Wilson (2001) set up a mathematical program in order to take an optimum mix of the possible response actions holding, expressing (also known as skip stop) and short-turning. Yin et al. (2017) design an integer linear programming model to identify the best possible ways to bring backup trains into service. Notice that the response rules that have been considered within these studies, in principle can be applied to circle lines in practice, too.

Furthermore, Li et al. (2015) presented a robust model predictive controller to regulate the operation of trains under uncertain disturbances caused by passenger fluctuations. Moreover, Li et al. (2017) investigated the joint optimization of the train regulation and passenger flow control for the busy metro lines to improve the headway regularity. In addition, Wang et al. (2019) designed an automatic train regulation algorithm to cope with the small disturbances by adjusting the running and dwell times of the involved trains, where a good trade-off among train delays, energy consumption and headway regularity is achieved.

In the case of more severe disruptions, e.g., the blockage of a track section or a station for 20-30 minutes, a combination of train dispatching measures is required as given in Puong and Wilson (2008); Cadarso et al. (2013), Xu et al. (2016) or Gao et al. (2016). The response rules that they are considering include short-turning, stop-skipping, cancellation, re-timing, and adding standby trains.

Specifically, Puong and Wilson (2008) investigated the holding (i.e., trains waiting at stations) and the short-turning strategies to reduce the effects on the service of passengers 
and train operations. Gao et al. (2016) proposed an optimization model to restore the train traffic after disruptions much more quickly via stop-skipping strategy (i.e., the stopping pattern of trains are optimized based on the passenger demand) and to reduce the number of stranded passengers faster for a metro line. Moreover, a metro train scheduling strategy by adding standby trains is considered in Yin et al. (2017) for heavily congested metro lines with the aim of alleviating waiting passengers from crowded platforms. For the disruptions caused by complete blockages, Chang et al. (2019) presented a mixed integer linear programming model to minimize the train delays and the cancellation of train services, where the available rolling stocks in the depot that could be put into operation are also considered.

Carrel et al. (2010) broaden their considerations to another aspect that is very important in the daily practice of delay management: "The reliability of the system is found to depend on many endogenous factors [...] Aside from the objective of maintaining adequate levels of service from an operations perspective and minimizing the impact of schedule deviations on passengers, considerations relating to management of crew and rolling stock, safety, and infrastructure capacity have a major influence on service control decisions." This is particularly true for train operations other than at the Grade of Automation 4 (GoA 4, no staff on board the train, unattended train operation, UTO).

Some alternative way to assess the complexity of delay management could be to set up an appropriate model in some railway operations simulation tool. Indeed, putting the delay scenario that we are considering into such a tool is viable. But from that initial delay on, standard simulation runs typically exploit running time supplements in order to catch up the schedules of the trains, and if any new primary delay is considered, then it is most often derived from some probabilistic distribution. In contrast, in practice, a certain development of an initial delay can be foreseen: The larger the gap between two subsequent trains, the larger the occupancy of the train and crowdedness of the platforms. Of course, both risk to extend dwell times immediately. This is also referred to as a "snowball effect" (Kunimatsu et al., 2009). We are aware of one dedicated simulation study that takes into account both, the train signalling system as well as dwell times that are depending on the number of passengers who board and alight (Kariyazaki et al., 2013). This one study investigates the effect of one response rule that we are considering, too (holding). Yet, there has not been taken into account the particular structure of circle lines, nor any more involved response rules as they are applied in practice for many networks worldwide.

The paper is organized as follows. After this introduction, we point out key differences between circular and non-circular metro lines. Moreover, we specify the delay scenario that the response rules, that we are going to collect in the sequel, shall be suited for. In Section 3, we present selected characteristics of the metro circle lines with whose experts we were able to have interviews. Hereafter, in Section 4 we present a description of each of the response rules that have been mentioned by the experts of the metro networks during our interviews. We report the results of these interviews in Section 5, before we finally draw some conclusions.

Notice that this paper's title "The Ring Never Relieves" is a generous translation proposed by Prof. Jörn Pachl, based on a German statement of a member of the response team at DB Netz AG, responsible for the Berlin ring lines S41/S42: "Der Ring schenkt einem nichts." 


\section{Characteristics of Circle Lines Relevant to Delay Management}

Having shortly reviewed several branches of research directions in particular for delay management for metro lines, in this section we first define the delay scenario that we are going to consider throughout this paper. Second, we collect some well-established delay management options as they apply in particular to non-circle lines. Finally, we motivate why for circle lines, further response rules need to be applied.

Notice that in principle most of the response rules that we are collecting in particular in Section 4 could be applied to non-circle lines, too. However, since there are further wellestablished - and simpler - delay management options available in the case of non-circle lines, those further and more complex response rules are applied less frequently to noncircle lines.

\subsection{Delay scenario}

The delay scenario that we are considering are somehow small delays that occur relatively often, and which are likely to not compensate on their own, but rather increase over time, such that response actions need to be taken.

Although in the remainder of this contribution the specific reason for the delay is of no importance, let us provide a few possible examples in order to guide the reader's mind to appropriate scenarios:

- Technical failure of one door of the train and the train driver has to get to that door in order to lock it

- Technical failure of one track-side signal for one train (no permanent failure)

- A passenger requires medical assistance

- A passenger uses an emergency brake and the train driver has to get to the specific brake, check that everything is in order and seal the emergency brake

- Incoming delay of a train from another line when entering the circle track

- etc.

Nota bene: After the reason for the delay has been resolved after a couple of minutes, the infrastructure of the entire circle line can be used without any restrictions.

Many of the initial disruptions that we listed above, often result in primary delays between 7.5 and 10 minutes. For a ring service that is operated at a scheduled headway of, say, 5 minutes, these delays thus are resulting in gaps of 12.5-15 minutes between two subsequent trains, i.e., 2.5 to 3 times the scheduled headway. In particular, if the standard level of occupancy that a train carries when operating at the standard headway of 5 minutes is scaled to 100 , then the first delayed train is likely to face a level of occupancy of 250300 .

In any case, when carrying more than twice as many passengers as usual during peak hours, it is very much probable that the train is not going to catch up the delay. Rather, the dwell times even risk to exceed their scheduled durations. The key reason for this are crowded spaces inside the train next to the doors (e.g. crash load of 4 passengers per $\mathrm{m}^{2}$ ). Depending on the train class, sometimes even slower acceleration could result and cause a further increase of the delay. Hence, such relatively small initial delays most probably do not disappear on their own, but rather risk to increase further.

\subsection{Well-established delay management options for non-circle lines}

Consider the non-circle metro line that is shown in Figure 1. The lines are the tracks for the two directions. And each grey box represents one train. In the spirit of a most simple cellular 
automaton (cf. Kariyazaki et al., 2017), when considering train movements and delays in the sequel, in our illustration it takes three time steps for a train to get to the current position of the preceding train. In particular, in our example, one complete turnaround in one of the terminus stations at the western and eastern end (from the arrival position to the departure position) is scheduled to take three time units, too.

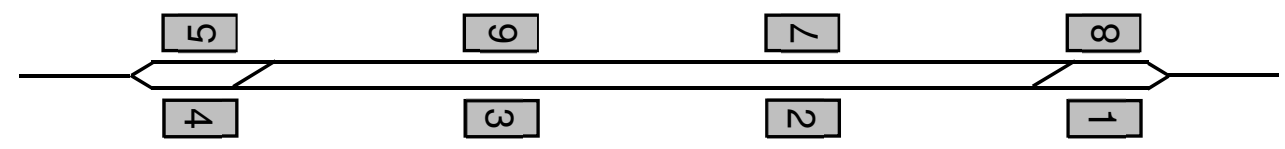

Figure 1: Scheduled operation on a non-circle line

Recall that along a non-circular line, often there are certain "natural" time buffers contained in the scheduled turnaround times. Moreover, sometimes even short-turning immediately at the platform can be an option, given that appropriate switches are located in front of the platform. In Figure 2 we illustrate the delay scenario in Part (a), where Train 1 faces a delay of 1.33 times the scheduled interval time. The gap thus results in seven time units compared to three during scheduled operation. Notice that the numbers within the train symbols refer to the identification numbers of the physical train sets, not to any logical train numbers as they might be defined in some schedule.

In Part (b) it is illustrated that Train 1 performs a short-turn immediately at the platform of the eastern terminus station without driving to the siding that is located right behind the platform. This is assumed to save two time units (cf. the reduced distance from Train 8 to Train 1 in Part (c)): one from omitting to drive to the siding, the other from from using the time buffer that is scheduled in the turnaround activity.

In Part (c) Train 2 has performed the "long" turnaround, i.e., by moving to the siding. But there, it consumed the scheduled time buffer of one time unit. In contrast, since Train 3 always meets its schedule (being the last train in the congestion), there is no need to consume its scheduled time buffer. Hence, a small gap from Train 2 to Train 3 reappears.

To summarize, if for a non-circular metro line there are certain time buffers contained in its scheduled turnaround times in the terminus stations, applying only very limited response action could bring the system back to schedule within a reasonable amount of time.

Another instrument that could be applied to such a delay scenario on a non-circle metro line is partial cancellation, provided that the track infrastructure admits trains to get onto the track of the opposite direction already a few stations prior to the terminus station. There, from a passengers' perspective, it could be preferable to let Train 1 and Train 2 change their positions by letting the crowded Train 1 continue until the terminus station, while the much less occupied Train 2 performs a short-turn a few stations earlier, hereby causing the partial cancellation. However, this results in changes to the crew and vehicle schedules that require to be managed in the sequel. 


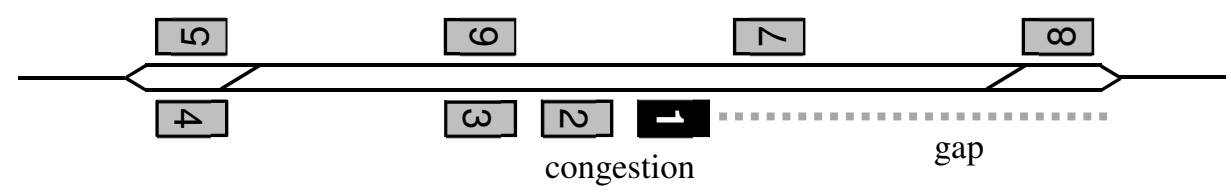

(a): Delay scenario and notation: train unit 1 faced a delay (1.33 x scheduled interval)

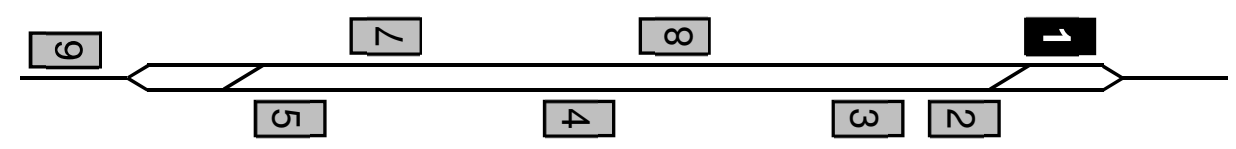

(b): Short turn of train unit 1 at platform of the east terminus station

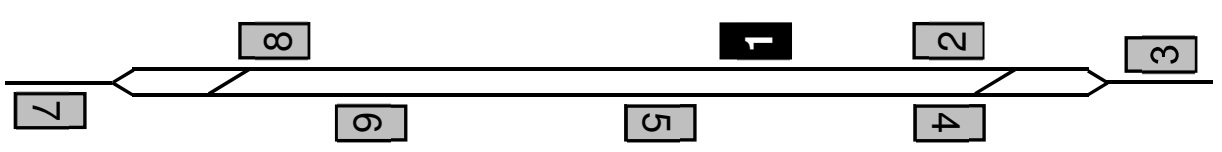

(c): Time buffer consumed by train unit 2 during turnaround in east terminus station

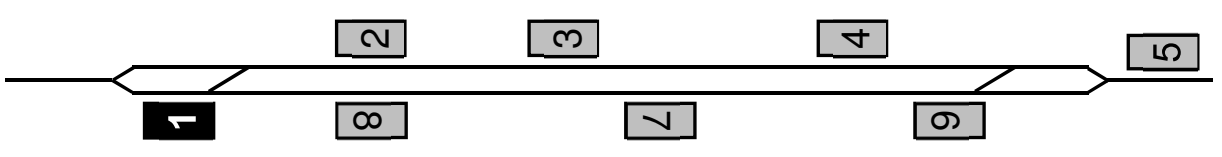

(d): Short turn of train unit 1 at platform of the west terminus station

Figure 2 (a-d): Using time buffers and applying short turning to a non-circle line

\subsection{Major goal of the response action for a circle line}

Now, let us turn to circle lines. In the absence of any terminus stations, there are not any scheduled time buffers available in any turnaround activities. In contrast, performing a full round along the circle, the train is in passenger service during $100 \%$ of the time, i.e., at the theoretical maximum of the efficiency that the asset "train" could have. In particular, there are no time buffers contained in any scheduled turnaround activities, there are not even any turnaround activities at all. This is why we are going to present and discuss tailored response rules for circle lines in Sections 4 and 5.

The delay scenario that we are using in the sequel to illustrate various response rules is depicted in Figure 3 (b). There, compared to the scheduled operation in Part (a), Train 1 faced an initial delay of 1.5 times the scheduled headway time, i.e., three time units when interpreting the model again as a most simple cellular automaton. This results in a gap of 2.5 times the scheduled headway time, or five time units. Right behind Train 1, further knock-on delays (aka secondary delays) are getting into effect for Trains 2 and 3. 


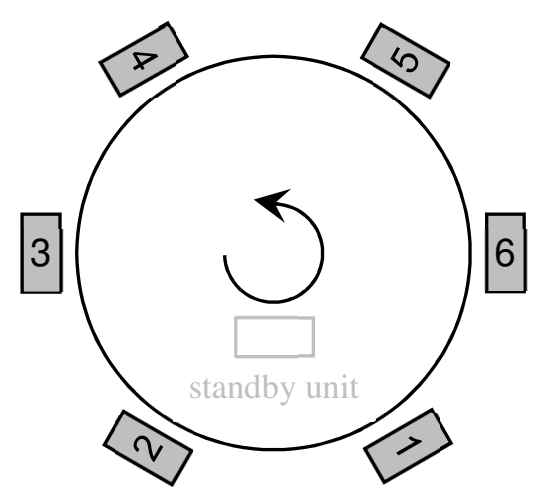

(a): Scheduled operation

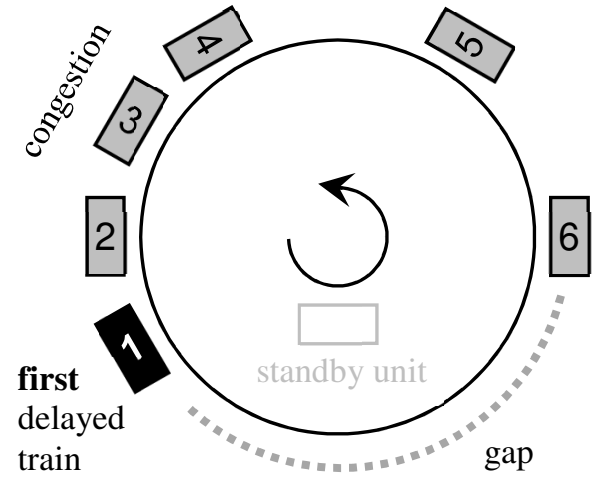

(b) Delay scenario and notation

Figure 3 (a-b): Scheduled operation compared to the delay scenario to be considered

In the absence of any "natural" time buffers in turnaround times, on a circle line, it cannot be expected that the delay is going to get compensated automatically, i.e., without any active response action. In contrast, due to the increasing crowdedness of Train 1, its delay is much likely to increase.

This is why it is not a big surprise that if there occurred a significant gap during the operations of a circle line, then the response teams of all the metro networks that we were in touch with put high priority on filling the gap. Some even emphasized that it is always a major concern for them to prevent any gap to arise. Otherwise, in particular for highly congested lines, the initial delay increases more and more just like a snowball (Kunimatsu et al., 2009).

We already indicate that one of the strategies that we are investigating is to schedule some standby unit in order to put it into operation to fill the gap. At first sight, this could appear somehow inefficient. But even when counting in the example of Figure 1 both arrival and departure in both terminus stations as being in passenger service, the efficiency of the trains of that line will not exceed $83.3 \%$. Notice that this level of efficiency is essentially comparable to the value of $85.7 \%$, which results for the circle line if in addition to the six trains in operation, there is scheduled one extra standby train unit.

\section{Circle Lines in Practice}

In our worldwide study, we are able to report on the dispatching experience with the circle lines of the following cities:

- $\quad$ Beijing (Lines 2 and 10), China

- Berlin, Germany

- Chengdu, China

- Copenhagen, Denmark

- Glasgow, United Kingdom

- Hamburg, Germany

- $\quad$ Madrid (Line 6), Spain 
- Moscow, Russia

- Seoul, South Corea

- Tokyo, Japan

- Zhengzhou, China.

First, we present certain properties and characteristics of all the abovementioned circle lines. These also include selected reference values that we computed based on publicly available information about the lines together with certain assumptions that have been made. Second, we present a few selected special features that are present in only one of the networks.

\subsection{General Properties of the Circle Lines}

The data in Table 1 are intended to briefly present the analysed circle lines and will be used to estimate the available buffer times. To begin with, notice the very different length of the lines. The shortest is $10.5 \mathrm{~km}$ long and was opened as early as 1896 in Glasgow while the longest lines can be found in emerging Asian megacities. This applies to Line 10 in Beijing with a length of $57.1 \mathrm{~km}$ as well as the $48.8 \mathrm{~km}$ long Line 2 in Seoul. Taking into account the number of stops shows that eight systems have average station distances above $1 \mathrm{~km}$, especially in Moscow even $1.6 \mathrm{~km}$. All Asian lines have platform doors (except for two stations in Tokyo) while in Europe this is only the case in Copenhagen.

The speed limit is almost always $80 \mathrm{~km} / \mathrm{h}$. It is lower only for the two oldest systems in Glasgow and Hamburg whereas in Tokyo it is $10 \mathrm{~km} / \mathrm{h}$ above. According to the schedule, the avarage speed for one round is between $26 \mathrm{~km} / \mathrm{h}$ and $42 \mathrm{~km} / \mathrm{h}$. Looking at the parameters presented so far it is not surprising that the lines in Glasgow and Hamburg can be found in the lower range and in Moscow at the upper range.

The train lengths in Copenhagen, Glasgow and Hamburg are more in the order of light rail. The trains in Seoul and Tokyo are about 200m long, and are thus longer than average. All values in Table 1 are rounded to integer multiples of five. Six systems have a uniform fleet. The routes of four circle lines are also used by trains breaking into the ring or leaving it. The percentage of the circle track that is used by non-circle lines is $100 \%$ in Hamburg, roughly $75 \%$ in Berlin and roughly $33 \%$ in Copenhagen. The level of automation is different. Most lines are operated at level GoA 1 (Grade of Automation 1: non-automated train operation), in Beijing there exists level GoA 2 (semi-automatic train operation) and only in Copenhagen one can find GoA 4 (unattended train operation).

The values in the column "average speed" refer to the scheduled time for one complete round of the circle line. The respective values are depicted in Table 2. The star (*) for Madrid is intended to highlight that the scheduled time for one round is ranging between 45 and 60 minutes during night service and peak hours, respectively, see Section 3.2. Similarly, the double star (**) for Hamburg refers to the special line concept, which is explained in Section 3.2, too.

In order to be able to classify the disposition measures on the individual lines, the available buffer times in terms of running time supplements are theoretically and roughly estimated in Table 2. For this purpose, the time spacing and the scheduled travel times of the lines during the peak hour were taken from many publicly available sources, although the two parameters do not exactly match in all cases (in principle, the scheduled time for one complete round should be an integer multiple of the scheduled headway between two subsequent trains). Based on the top speeds (Table 1) and accelerations (Table 2), we first compute a theoretical value for the estimated minimum running time (see the proceedings version for a more detailed description of the computation). Then, we add 20 s dwell time for each station along the circle line. Comparing the resulting sum with the scheduled time 
for one complete round, this provides a rough theoretical idea of the amount of buffer time that is contained in the scheduled time for one full round.

Table 1: Overview of selected properties of metro circle lines (except for time values)

\begin{tabular}{lccccccccc}
\hline City & Line & $\begin{array}{c}\text { len } \\
\mathbf{( k m})\end{array}$ & $\begin{array}{c}\text { number } \\
\text { of } \\
\text { stations }\end{array}$ & $\begin{array}{c}\text { top } \\
\mathbf{s p e e d} \\
(\mathbf{k m} / \mathbf{h})\end{array}$ & $\begin{array}{c}\text { avg } \\
\text { speed } \\
(\mathbf{k m} / \mathbf{h})\end{array}$ & $\begin{array}{c}\text { train } \\
\text { length } \\
(\mathbf{m})\end{array}$ & $\begin{array}{c}\text { uni- } \\
\text { form } \\
\text { fleet }\end{array}$ & $\begin{array}{c}\text { other } \\
\text { lines on } \\
\text { circle }\end{array}$ & GoA \\
\hline Beijing & 2 & 23.1 & 18 & 80 & 31.5 & 115 & + & - & 2 \\
Beijing & 10 & 57.1 & 45 & 80 & 32.9 & 115 & - & - & 2 \\
Berlin & S41/42 & 37.1 & 27 & 80 & 37.1 & 145 & - & + & 1 \\
Chengdu & 7 & 38.6 & 31 & 80 & 35.1 & 140 & + & - & 1 \\
Copenhagen & M3 & 15.5 & 17 & 80 & 31.0 & 40 & + & + & 4 \\
Glasgow & N/A & 10.5 & 15 & 54 & 26.3 & 40 & - & - & 1 \\
Hamburg & U3 & 17.5 & 23 & 70 & 26.3 & 60 & - & $+* *$ & 1 \\
Madrid & 6 & 23.5 & 28 & 80 & $27.1 *$ & 110 & - & - & 1 \\
Moscow & 5 & 19.4 & 12 & 80 & 41.6 & 130 & + & - & 1 \\
Seoul & 2 & 48.8 & 43 & 80 & 34.9 & 195 & - & + & 1 \\
Tokyo & Yama & 34.5 & 30 & 90 & 34.5 & 215 & + & - & 1 \\
& note & & & & & & & & \\
Zhengzhou & 5 & 40.7 & 31 & 80 & 33.9 & 140 & + & - & 1 \\
\hline
\end{tabular}

Table 2: Overview of selected time values of metro circle lines (scheduled values as well as computed reference values)

\begin{tabular}{|c|c|c|c|c|c|c|c|c|c|}
\hline \multirow[t]{2}{*}{ City } & \multirow[t]{2}{*}{ Line } & \multirow{2}{*}{$\begin{array}{c}\text { peak } \\
\text { inter- } \\
\text { val } \\
(\mathrm{min})\end{array}$} & \multirow{2}{*}{$\begin{array}{l}\text { time } \\
\text { sche- } \\
\text { duled } \\
(\text { min) }\end{array}$} & \multirow{2}{*}{$\begin{array}{l}\text { estimated } \\
\text { minimum } \\
\text { running } \\
\text { time (min) }\end{array}$} & \multirow{2}{*}{$\begin{array}{c}\text { accele- } \\
\text { ration } \\
\left(\mathrm{m} / \mathrm{s}^{2}\right)\end{array}$} & \multirow{2}{*}{$\begin{array}{c}\text { decele- } \\
\text { ration } \\
\left(\mathrm{m} / \mathbf{s}^{2}\right)\end{array}$} & \multicolumn{3}{|c|}{ approx. buffer time (\%) } \\
\hline & & & & & & & $\begin{array}{c}\text { dwell } \\
\text { time } \\
30 \mathrm{~s}\end{array}$ & $\begin{array}{c}\text { dwell } \\
\text { time } \\
20 \mathrm{~s}\end{array}$ & $\begin{array}{c}\text { dwell } \\
\text { time } \\
15 \mathrm{~s}\end{array}$ \\
\hline Beijing & 2 & 2.5 & 45 & 27.6 & 0.8 & 0.7 & 18.7 & 25.4 & - \\
\hline Beijing & 10 & 2 & 104 & 68.4 & 0.8 & 0.7 & 12.6 & 19.8 & - \\
\hline Berlin & $\mathrm{S} 41 / 42$ & 5 & 60 & 41.5 & 1.0 & 0.7 & 8.3 & 15.8 & - \\
\hline Chengdu & 7 & 3.5 & 66 & 46.6 & 0.8 & 0.7 & 5.9 & 13.7 & - \\
\hline Copenhagen & M3 & 3 & 30 & 18.9 & 1.1 & 0.9 & 8.7 & 18.1 & \\
\hline Glasgow & N/A & 4 & 24 & 15.7 & 1.1 & 0.9 & 3.5 & 13.9 & - \\
\hline Hamburg & $\mathrm{U} 3$ & $2 ; 3 ; 5$ & 40 & 25.0 & 1.0 & 0.7 & 8.8 & 18.4 & - \\
\hline Madrid & 6 & $2-4$ & 52 & 33.6 & 0.8 & 0.7 & 8.5 & 17.5 & - \\
\hline Moscow & 5 & 1.75 & 28 & 21.4 & 0.8 & 0.7 & - & 9.4 & 12.9 \\
\hline Seoul & 2 & 2.33 & 84 & 58.6 & 0.8 & 0.9 & 4.7 & 13.2 & - \\
\hline Tokyo & $\begin{array}{c}\text { Yama } \\
\text { note }\end{array}$ & 2.33 & 60 & 42.7 & 0.8 & 0.7 & 3.9 & 12.2 & - \\
\hline Zhengzhou & 5 & 5.5 & 72 & 48.2 & 0.8 & 0.7 & 11.6 & 18.7 & - \\
\hline
\end{tabular}

The buffer times that we determined this way have a surprisingly small spread and are around $12-19 \%$ of the scheduled travel time. The only outliers are Beijing (larger buffer times) and Moscow (shorter buffer times). For the latter, we also indicate the buffer percentage value that results when assuming dwell times of $15 \mathrm{~s}$ only. For all the other circle lines we also provide the resulting buffer percentage values when assuming dwell times of 30 s in each station. For the first delayed train behind a gap (see Figure 3 (b)) dwell times of 
30s might even be challenging to attain. In particular buffer times below $9 \%$ support the need for appropriate response action, because the first delayed train might not be likely to catch up its delay on its own.

Notice that we might have been interested in many further properties of the networks. Yet, it was beyond our study to identify them, essentially for two reasons: Some of them might have caused significant investigation efforts (e.g. detailed values of key performance indicators), while others might have been considered as business secrets (e.g. detailed track map with each crossover, siding etc., exact values for the punctuality, detailed rules according to which the drivers' shifts must be planned).

\subsection{Special Features}

In this section, we present selected features and operating schemes of some of the circle lines.

\section{Berlin}

Actually, the circle line in Berlin is not any true metro line: officially, they are railway lines. However, from a passenger perspective, a metro-like service is offered, i.e., short intervals and a much higher average speed than that of tramways. Yet, due to the legal framework in Germany, there are two response teams responsible for managing the operations of the circle line: one belonging to the infrastructure manager (DB Netz AG), the other belonging to the train operating company (railway undertaking, S-Bahn Berlin $\mathrm{GmbH}$ ). In particular, the latter is responsible for scheduling the resources such as train units and train drivers. We performed two separate interviews with representatives of the two teams, where the answers have been highly compatible to each other.

For sake of completeness, let us put together two values from the previous tables. In Table 2, we mention a headway of $5 \mathrm{~min}$ for the actual circle lines. But in addition, according to Table 1, there are further lines operating on the circle track. On roughly $50 \%$ of the circle track, there are six trains within 20 minutes, on some parts even seven trains.

\section{Hamburg}

In Hamburg, being the second largest city in Germany, Line U3 operates as follows: Wandsbek-Gartenstadt - Barmbek - Central Station - St. Pauli - Barmbek (see Figure 4). During peak hours, in both directions there is a train every five minutes. Thus, this is not a continuous full-ring service. However, in addition to these trains, there is another train every 10 minutes, which is operating as a full circle line service for several rounds in a row: Barmbek - Central Station - St. Pauli - Barmbek, both clockwise and counter-clockwise. One such round takes 40 minutes.

In the case of delays, in Wandsbek, the scheduled turnaround time can be reduced by about 5-6 minutes. Hence, for these trains it is possible to catch up a certain level of delay in the spirit of Figure 2. Let us summarize some key properties that result from this line concept:

- Each segment of the full circle service is also served by the non-circle trains from and to Wandsbek.

- If there is a delay on, e.g., the clockwise track, then the trains from Wandsbek are much likely to enter the circle track without any delay, thus preventing larger gaps to persist for more than one round.

To summarize, at first sight the mixture of the trains from and to Wandsbek and the full circle trains could appear a bit complicated. Yet, it turns out that at least to some extent "filling the gap" comes kind of automatically about every five minutes. 


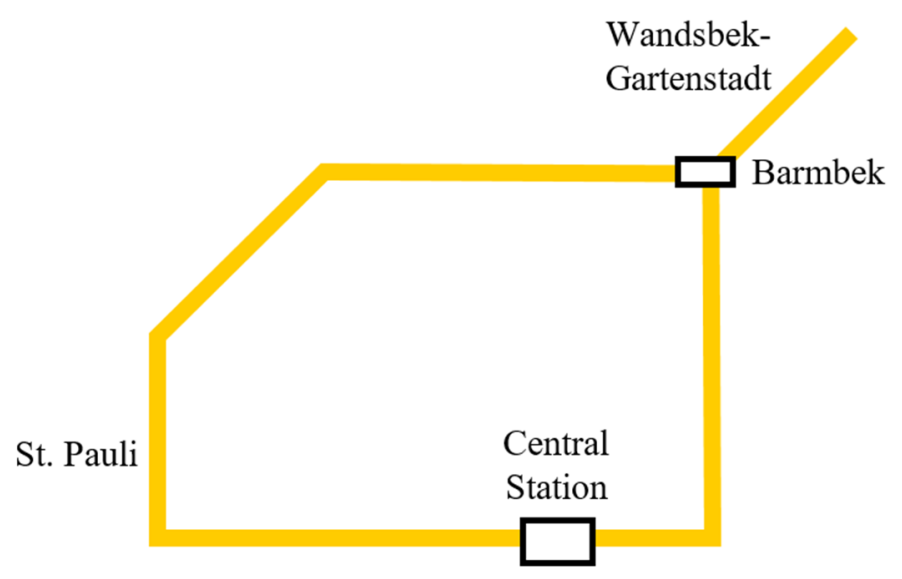

Figure 4: Line map of Line U3 in Hamburg with full circle operation Barmbek - Central-Station - St. Pauli - Barmbek

\section{Madrid}

The metro of Madrid is the only one that reported to us to have scheduled a significant amount of running time supplement during peak hours. While the full Line 6 can be operated in 45 minutes during the night service, the time that is scheduled during peak hours for one round in the counter-clockwise direction is not less than 60 minutes - hence a running time supplement of $33 \%$.

Of course, this is motivated by a much larger number of passengers, resulting in very high occupancies of the trains as well as crowded platforms. These require immediately longer dwell times in the stations. Such a time buffer can be discussed from various perspectives, e.g.:

- The part of the running time supplement of $33 \%$ that is not immediately necessary for the longer dwell times at the platforms might make it more easy to catch up for at least a certain part of delays, by operating as fast as possible.

- Given a fixed interval between any two trains that is necessary to transport all the passengers, increasing the scheduled time for one round immediately requires an additional number of train units (as well as train drivers), which could be considered inefficient.

- Comparing this circle line operation to other non-circular lines reveals that also there, in addition to the immediate time that is required to operate between the two terminus stations, typically there are some extra trains part of the schedule, in particular for the time that is spent in turnarounds at the endpoints - hence, parts of the above inefficiency could be considered to be somehow "normal".

- Yet, one could ask the following: Could it maybe provide more flexibility in responding to delays, when refraining to add the "last" $1-2$ of these extra train units to the scheduled service but to keep them as backup (standby) train units instead, in order to flexibly fill possible gaps? 


\section{Response Rules for Circle Lines}

Recall from Section 2.3 that in the case of the delay scenario that we are considering, the priority of the response action is on "filling the gap". Hence, in this section we are collecting several different techniques of how to fill a gap that occurred on a circle line. These response rules are listed increasingly according to their conceptual complexity:

- $\quad$ accelerating the first delayed train (expressing / skip stop, taking a shortcut),

- slowing down all the trains of the affected direction of the circle line (holding),

- inserting a dedicated standby (backup) train unit or

- inserting another train unit by taking it from some other regular service (from the opposite direction of the circle line, from another line, or immediately from the delayed direction of the circle line).

Observe that this order does not correlate with an increasing need to involve extra resources, or at least an increasing need to modify the schedules of certain resource.

For each response rule, in addition to its description, we mention their key preconditions for that they are applicable at all. Moreover, the description of some of the rules will be supported by selected figures. Within these, recall from Figure 1 that the numbers within the train symbols refer to the identification numbers of the physical train sets, not to any logical train numbers as they are defined in some schedule. In addition, in Section 4.8 we finally sketch the impact that applying some response rule has on the schedules of train units and train drivers, as well as on passengers (challenges in passenger information, need to change a train).

Several of these response rules will add an extra train unit to the delayed direction of the circle line in order to fill the gap, be it from the opposite direction, from another line, or from a backup, or, standby unit. Whichever source such an extra train unit has, operators are always aware of the following general rule of thumb: Whenever an additional train unit is inserted, another train unit has to be taken out of service in order to end up with the scheduled number of train units in service. This is for two reasons: First, in particular if the extra train unit has been taken from the opposite direction or even from another line, then the gap that is resulting there must be filled as soon as possible. Second, operating more trains than scheduled on the circle line potentially could risk to cause congestions in its own.

Another general point are the procedures that are necessary in order to remove a train from its scheduled service. In the first case, when taking some train from the opposite direction and it thus shall perform a turnaround right at the platform in order to continue into the delayed direction, then the time that it takes for the train driver to get to the driver's cabin at the other end of the train has to be considered carefully, unless a standby driver could replace the arriving train driver. If the walking time would take too much time, then the entire operation risks to be considered being infeasible. In the second case when it shall move to a siding first, there could be a certain amount of time for all the passengers to get off the train - if this is necessary - and to check that nobody has left in the train. There are at least three preconditions under which this could be feasible:

- The train driver is assisted by some other staff member.

- The train driver can check the emptiness of the train from the driver cabin by using a video surveillance system.

- The train could be parked on a track with a platform where it does not block any subsequent train.

Otherwise, if none of these three preconditions apply, such an operation could take too much time, block the subsequent trains and is therefore not part of the "toolbox" of the 
response team.

\subsection{Expressing (also referred to as skip stop)}

Fill the gap by speeding-up the first delayed train in order to catch up its delay and thus reestablish the scheduled headway to its predecessor. To this end, let the first train behind the gap omit several scheduled stops in order to catch up its schedule. The same is then applied for the subsequent trains in the congestion.

\section{Key Preconditions}

- Sufficiently high gain in time, i.e. relatively moderate speed limits when passing at a platform (preferably the platforms are equipped with doors/gates, and these do not impose any speed restrictions due to possible air pressure effects)

- Effective passenger information in particular on board the trains that are about to skip a stop. Moreover, all the passengers need to show a certain level of cooperation for that those passengers, who want to leave the train as a consequence of announcing subsequent stops to be skipped, can do so.

4.2 A train unit from the same delayed direction of the circle line performs a shortcut Fill the gap by letting a train from the congestion perform a shortcut apart from the circle line, see the corresponding figure in the proceedings verion. This way, the train can then be inserted into the gap, and continue in the initial schedule of the first delayed train.

\section{Key Preconditions}

- Presence of a shortcut line that is at least roughly twice the scheduled interval time faster compared to riding the full round of the circle

- Sufficient amount of residual capacity for carrying one extra train trip

- Authorization of the train driver of the circle line to drive also on the shortcut line

- A certain degree of flexibility in both, the vehicle schedule and the crew schedule, because the Trains 1, 2 and 3 - together with their train drivers change their positions in the schedule

Notice that in principle, the track of the opposite direction could serve as the shortcut, too. Then, the applicability of this response rule relies on crossovers and/or sidings at appropriate positions. Recall from the discussion at the beginning of this section that time consuming procedures could risk to apply in such a case.

\subsection{Holding}

Fill the gap by slowing down the last trains before the gap (Train units 4, 5, and 6 in Figure 5 (b)). Delay them voluntarily, e.g. by holding them longer than scheduled in their present station, such that the gap to the first delayed train decreases to the scheduled headway (compare the gap from train unit 6 to train unit 1 in Part (c) to the initial gap between the same two train units in Part (a)). As an immediate consequence, hereafter formally all trains will operate behind the schedule, see Figure 5. By the way, an appropriate way to think of this response rule might be that of a "paternoster". 


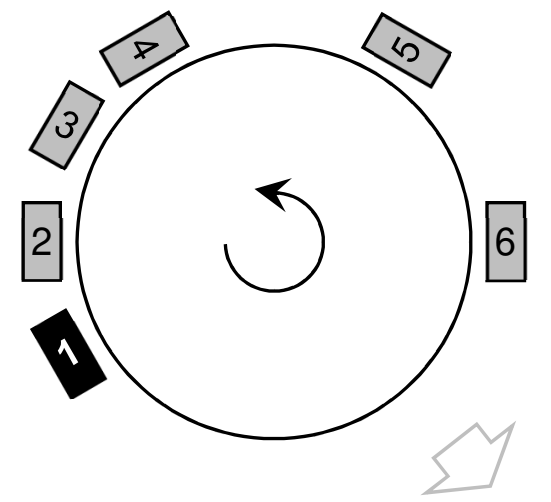

(a): Initial delay scenario

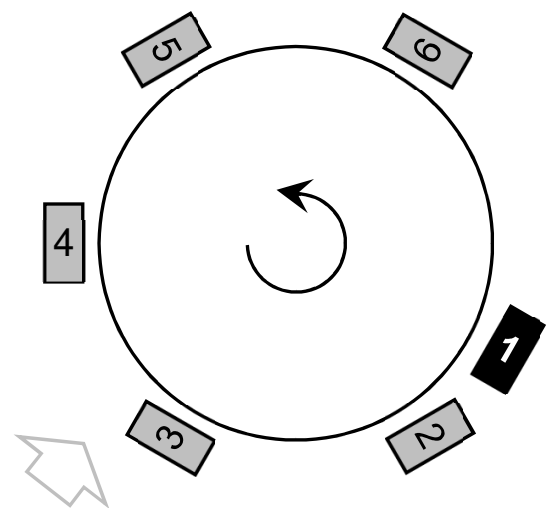

(c) (almost) balanced service

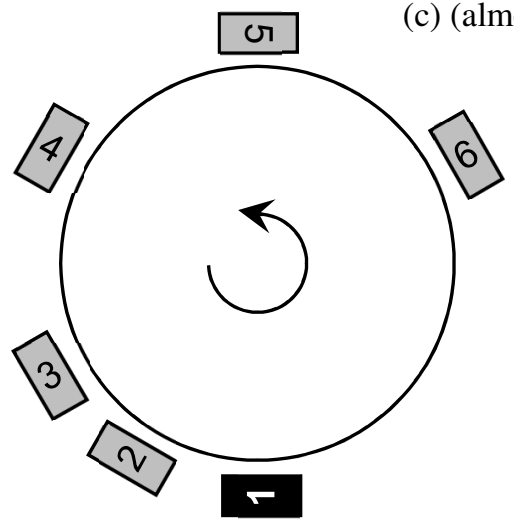

(b): Train unit 4 operates slower than scheduled in order to increase its headway to train unit 3 - so do train units 5 and 6 , to keep their scheduled headways

Figure 5 (a-c): Illustration of the "holding" response rule - Type 1

\section{Key Preconditions}

- Section of the circle line which is not shared with any other non-circle line, where the trains of no other non-circle line would be delayed, while trains of the circle line wait for a couple of minutes

- A certain understanding of the passengers using the initially punctual trains, why these are operating slower than the passengers might be used to

- Very high flexibility in both, the vehicle schedule (in order to still get the right trains to a maintenance site) and the crew schedule (in order to ensure break times as they are likely to be required by law), because at the very end of applying this response rule, the schedules of all trains and train drivers are shifted essentially by the initial delay. Alternatively, if there are neither any strict punctuality measures that the company has to conform to for its trips during the remainder of the day, nor any severe disadvantages of letting all the train drivers terminate their work by, say, 10 minutes later, then the measurable 
operational delay of all the trains simply could be kept until the end of the day, while the passengers might experience an on-time service.

\subsection{Inserting a standby train unit}

Fill the gap by putting a standby train unit (backup train unit) into service right before the first delayed train, see the proceedings version. In turn, take another train unit of the delayed direction (somewhere shortly behind the first delayed train, e.g. in the congestion, where trains tend to be filled below average) out of service in order to resolve the congestion.

\section{Key Preconditions}

- Standby train unit together with a standby train driver available, ideally located shortly behind the location where the "gap" occurred first

- Sufficiently fast processes to take a train unit out of the congestion without blocking the subsequent trains too long in order not to create a new "gap", recall the discussion at the beginning of this section. For this particular response rule, alternatively it could be sufficient to reach a spare track with a platform in some nearby station that is not part of the circle line and let the train wait there (or in a siding over there)

- A certain degree of flexibility in both, the vehicle schedule and the crew schedule, because because the Trains 1,2 and 3 - together with their train drivers - change their positions in the schedule

- Sufficient (monetary) incentive such that it pays off to schedule a standby unit, which finally could remain unused during several days of a week

\subsection{Inserting a train unit from the opposite non-delayed direction of the circle line}

Fill the gap by letting two trains of the two opposite directions of the full circle line change their directions such that the train of the non-delayed direction is inserted right before the first delayed train. In turn, the train of the delayed direction leaves the congestion (somewhere shortly behind the first delayed train).

\section{Key Preconditions}

- Availability of a pair of crossover tracks (switches) to get from the delayed direction of the circle line to track of the non-delayed direction of the circle line and vice versa (ideally, the distance between the two crossover tracks of this pair should be in a distance of 2-3 times the scheduled interval time)

- If there are no immediate crossover tracks but the trains must go to a siding first, then the discussion of time-consuming processes to get trains out of passenger service from the beginning of this section risks to apply.

- Effective passenger information in particular on board the trains that are not about to continue to their final destinations, but continue on other routes.

- Interchangeable types of train units between the two opposite directions

- Authorization of the train driver of one direction of the circle line to drive also on the opposite direction of the circle line (or some appropriate standby train drivers being available)

- A certain degree of flexibility in both, the vehicle schedule and the crew schedule, because the two involved train units and train drivers change their positions in the schedule 


\subsection{Inserting a train unit from another non-circle line}

Fill the gap by taking a train unit of that other non-circle line and let it continue on the full circle line right before the first delayed train. In turn, take another train unit of the delayed direction out of the congestion (somewhere shortly behind the first delayed train) and put it to the other line.

\section{Key Preconditions}

- Switches to get from a non-circle line to the circle line and vice versa

- Effective passenger information in particular on board the trains that are not about to continue to their final destinations, but continue on other routes.

- Interchangeable types of train units between the two lines

- Authorization of the train driver of the circle line to drive also on the non-circle line and vice versa (or some appropriate standby train drivers being available)

- A certain degree of flexibility in both, the vehicle schedule and the crew schedule, because the two involved train units and train drivers change their positions in the schedule

\subsection{Inserting a train unit from the same delayed direction of the circle line}

Fill the gap by taking one or two trains of the delayed direction out of the congestion (somewhere shortly behind the first delayed train, see Figure 6 Parts (b) and (c)) and inserting them in "the next round" into the gap, i.e. in front of the first delayed train, see Train 2 in Part (d).

\section{Key Preconditions}

- Free track (siding or at platform, ideally shortly behind the location where the "gap" occurred first) to let a train unit out of the congestion wait until the "gap" is getting back to this location after having moved one round along the circle (in between train units 6 and 1 in Part (a))

- Sufficiently fast processes to take a train unit out of the congestion without blocking the subsequent trains too long in order not to create a new "gap", recall the discussion at the beginning of this section. For this particular response rule, alternatively it could be sufficient to reach a spare track with a platform in some nearby station that is not part of the circle line and let the train wait there (or in a siding over there)

- A certain degree of flexibility in both, the vehicle schedule and the crew schedule, because the Trains 1 and 2 - together with their train drivers - change their positions in the schedule

Notice that in principle it would increase flexibility to have the ability to park a train at a platform on a track where it does not block the regular service. Yet, it does not resolve a key challenge with respect to rescheduling the train drivers in the course of this response rule: Comparing Parts (a) and (d) in Figure 6, the train drivers of train units 1 and 2 changed their positions in the schedules. The same holds for the train units themselves. In order to bring them "back to schedule", the easiest way would be to have them in the same station at a time and then switch back their identities. However, from the four parts of Figure 6 it is getting clear that these two train units and train drivers will never meet in the same station. Hence, the described infrastructure does not support such an easy switch back. What is 
happening, is simply a kind of overtaking something without catching it up ${ }^{1}$.

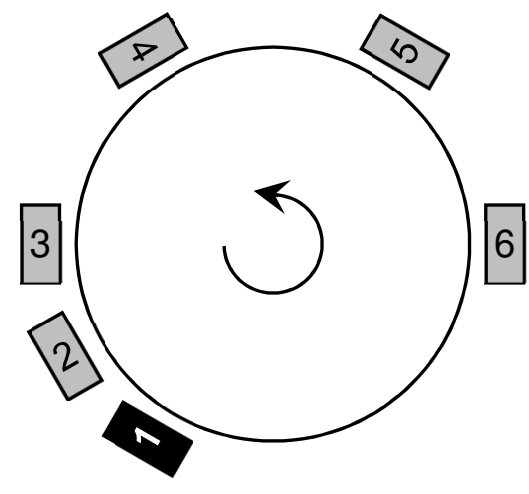

(a): Initial delay scenario

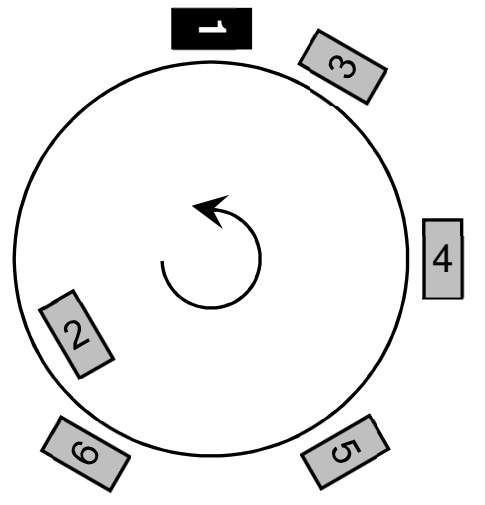

(c): ... waiting for the gap to get back

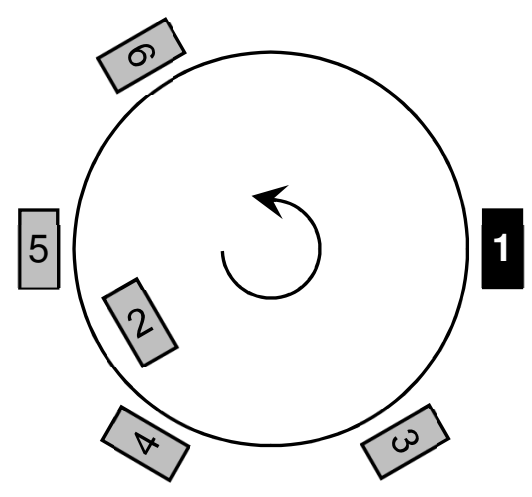

(b) Let train unit 2 wait in a siding...

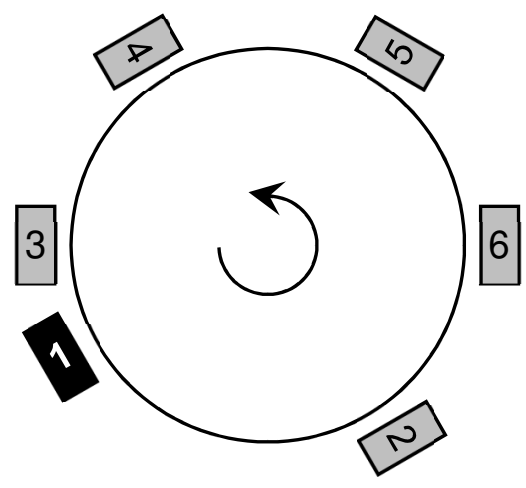

(d) (almost) balanced service

Figure 6 (a-d): Illustration of the response rule to insert a train unit from the same delayed direction of the circle line

\subsection{Overview of key features of the response rules}

On the one hand, the need for extra resources or a particular topology of the network is intrinsic to the respective response rules. On the other hand, we collect the impact that the seven response rules, which we presented, have with respect to passengers and resource schedules and provide an overview in Table 3.

Table 3: Overview of the impact that the response rules have on passengers and on the scheduled resources

\footnotetext{
${ }^{1}$ In German: „überholen ohne einzuholen“, a prominent political saying in the former German Democratic Republic since the late 1950s.
} 


\begin{tabular}{|c|c|c|c|c|c|c|c|}
\hline Impact on... & $\begin{array}{c}4.1 \\
\text { Skip } \\
\text { stop }\end{array}$ & $\begin{array}{c}4.2 \\
\text { Short- } \\
\text { cut }\end{array}$ & $\begin{array}{c}4.3 \\
\text { Hol- } \\
\text { ding }\end{array}$ & $\begin{array}{c}4.4 \\
\text { Stand- } \\
\text { by unit }\end{array}$ & $\begin{array}{c}4.5 \\
\text { Oppo- } \\
\text { site }\end{array}$ & $\begin{array}{c}4.6 \\
\text { Other } \\
\text { Line }\end{array}$ & $\begin{array}{c}4.7 \\
\text { Same } \\
\text { Direction }\end{array}$ \\
\hline $\begin{array}{l}\text {... passengers (get } \\
\text { off their train, } \\
\text { complexity of info) } \\
\ldots \text { schedules for train } \\
\text { units and drivers }\end{array}$ & 0 & 0 & ○* & 0 & 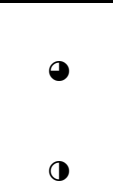 & - & 0 \\
\hline
\end{tabular}

*) Even though no passenger will have to get off a train in order to arrive at the intended station, passengers might expect to receive some information why their trains are being held and thus delayed.

Notice that we refrained from using the $100 \%$ Harvey ball in the row of how severe passengers might be affected by any of the response rules. The most severe case would be if the first train - which is likely to be occupied close to its crash load (e.g. 4 passengers per $\mathrm{m}^{2}$ ) - would not serve any of the stations where passengers might want to get off: Applying the Expressing rule ("skip stop"), the highest occupied train unit is concerned, but some of its passengers will reach their destination while staying on this train. In turn, taking some train unit from an undisturbed service (be it from the opposite direction or a different line), the passengers of this other service have to leave their train - plus those who are on the (relatively empty) train that in turn will be taken out of the congestion of the delayed direction of the circle line in exchange.

Moreover, using the $100 \%$ Harvey ball in the row describing the impact on the schedules for train units and train drivers we assume that all the trains of the delayed direction will not stick to their scheduled train numbers and then continue all with the same amount of delay, but that the train numbers might rather be shifted by one or two intervals.

Finally, in order to apply "holding" as a response rule it may be considered as a precondition that trains need to be operated driverless or that the employment of the train driver staff must be very flexible. Flexibility may address for instance the following issues:

- ability to let a train driver in charge of a train for, say, half of its shift

- absence of the need to define at the beginning of the work fixed time windows within which certain breaks must take place

- $\quad$ possibility to replace one long break (e.g. 30min) with several shorter breaks

- possibility to extend the end of a shift by, say, one round of the circle

- $\quad$ possibility to replace the station where a shift is scheduled to end with another station (at no extra cost)

- no dependency of any train driver from any other line during a shift of the circle line

- availability of several of standby train drivers.

As usual, of course most of these facets of flexibility are in contrast to the comfort and in particular to the reliability of the working times of each individual train driver. In the absence of several of these features of flexibility, other response rules might be even more worthwhile to consider.

\section{Expert Interviews on Response Rules for Circle Lines}

After we collected seven response rules that are suited for the special case of metro circle lines, in this section we are going to present our second main contribution, i.e., an overview 
of the answers that we received from expert staff of the operators of twelve circle lines worldwide. A major goal was to find out which of the response rules are applied on a regular basis in which particular network. In general, the interviews had been carried out in such a way that at least one member of the authors' team had an online appointment with a member of the response team. Other modes are listed below:

- Chengdu, Moscow, and Zhengzhou

The local experts filled questionnaires that the authors were sending to them.

- Seoul

The answers were kindly provided to the authors by Sukmun Oh from the Korean Railway Research Institute.

First of all, let us stress that there was an immediate agreement on the priority of "filling the gap" in the interviews with each of the cities. In Table 4 we provide an overview, which of the response rules is how much likely to be applied in the case of disruptions on each of the circle lines. Also here, we are using a filled Harvey ball, whenever a response rule is applied relatively often. Yet, the fill levels are not to be understood to indicate any wellquantifiable probabilities of their likeliness for being applied. Hence, most often the "sum" of the Harvey balls in a row does not yield $100 \%$.

For sake of completeness, let us mention that none of the experts of the metro networks reported of any automated software system being in use for disruption management, even in the case of the fully-automated unattended GoA 4 service in the city of Copenhagen. This supports the relevance of our collection of response rules in order to ensure the best possible service for the passengers.

Table 4: Overview, how often the response rules from Section 4 are applied for the various circle lines

\begin{tabular}{|c|c|c|c|c|c|c|c|c|}
\hline City & Line & $\begin{array}{c}4.1 \\
\text { Skip } \\
\text { stop }\end{array}$ & $\begin{array}{c}4.2 \\
\text { Short- } \\
\text { cut }\end{array}$ & $\begin{array}{c}4.3 \\
\text { Hol- } \\
\text { ding }\end{array}$ & $\begin{array}{c}4.4 \\
\text { Stand- } \\
\text { by unit }\end{array}$ & $\begin{array}{c}4.5 \\
\text { Oppo- } \\
\text { site }\end{array}$ & $\begin{array}{c}4.6 \\
\text { Other } \\
\text { Line }\end{array}$ & $\begin{array}{c}4.7 \\
\text { Same } \\
\text { Direction }\end{array}$ \\
\hline Beijing & 2 & 0 & 0 & 0 & 0 & 0 & $\bigcirc$ & 0 \\
\hline Beijing & 10 & 0 & 0 & ( & 0 & (1) & 0 & O \\
\hline Berlin & S41/42 & 0 & 0 & 0 & (1) & 0 & 0 & (1) \\
\hline Chengdu & 7 & $\bigcirc$ & $\bigcirc$ & $\bigcirc$ & 0 & $\bigcirc$ & $\bigcirc$ & $\bigcirc$ \\
\hline Copenhagen & 3 & 0 & 0 & 0 & 0 & 0 & 0 & 0 \\
\hline Glasgow & N/A & 0 & 0 & 0 & 0 & 0 & 0 & 0 \\
\hline Hamburg* & $\mathrm{U} 3$ & 0 & 0 & 0 & 0 & () $*$ & (1) & * \\
\hline Madrid & 6 & 0 & 0 & 0 & 0 & 0 & 0 & 0 \\
\hline Moscow & 5 & 0 & 0 & (1) & ( & (1) & 0 & (1) \\
\hline Seoul & 2 & 0 & 0 & 0 & 0 & 0 & 0 & 0 \\
\hline Tokyo & Yama. & 0 & 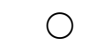 & 0 & 0 & 0 & 0 & $\bigcirc$ \\
\hline Zhengzhou & 5 & 0 & 0 & 0 & 0 & 0 & 0 & 0 \\
\hline
\end{tabular}

*) Recall the operating scheme in Hamburg from Section 3.2: There are always two Wandsbek trains in between two consecutive full circle trains. Although these trains are part of Line U3, too, operationally these may be considered as trains of another line, which regularly even change their direction relatively to the, say, clockwise full circle trains.

There are a few results that we want to highlight. On the one hand, there are three response rules that are applied somehow rarely. First, it is obvious that in the absence of the infrastructural preconditions, response rules such as using a shortcut or inserting a train 
from another non-circle line simply cannot be applied. For instance, only in the case of Berlin there had been reported a possible shortcut track (Halensee - Central Station Treptower Park) - and also there, it is not among the response rules that are applied most often. Second, it is striking that expressing is a response rule of choice only for the Beijing circle lines.

On the other hand, the two response rules that seem to be applied most often are inserting a standby train unit, and in particular holding the entire system in a paternoster way. The benefit and ease of use are very much clear for inserting a standby train unit. Yet, this comes at a certain cost, because the efficiency of the standby unit is very limited, of course. Nevertheless, together with the train units that are operating "endlessly" during the day, even when adding one or two standby train units, typically the efficiency of this fleet is not significantly less than the one that results from turnaround activities of non-circle lines. Regarding holding, given that the degree of flexibility in both, the schedules of the train units and the train drivers is sufficiently high, then it is a very effective and conceptually simple response rule. The Glasgow subway team expects this response rule to become applicable even more easily, as soon as their service will have been migrated to unattended operations (GoA 4), probably after the year 2025. But holding comes at a price, too: Since each train needs to be delayed, in the end many passengers are facing delays, see below.

In addition, let us shortly discuss response rule 4.7 , which is only applied on a regular basis for the Berlin ring Lines S41/42, and sometimes in Beijing. In particular, in Berlin, indeed it turns out to be a highly efficient response rule. Due to a lack of both, a spare track at a platform in some station of the circle line and a video surveillance system inside today's coaches, it is common practice that a train of the congestion of the delayed direction of the circle line (i.e. train unit 2 in Figure 6 (b)) is redirected to another station that is not part of the circle line (e.g. Charlottenburg, Schöneweide). There, it is likely that only few passengers have to get off the train, because most passengers might have alighted in the last station before leaving the circle line, and in general trains in the congestion tend to be much less crowded than the first delayed train. Then the train unit waits either at a platform or in a siding. Finally, when returning to the circle line in the next "round", since it is inserted right into the gap, typically there is a sufficiently high amount of time and free capacity in order to synchronize into the best possible position. In total, after two rounds (i.e. two hours in Berlin), most often the entire service of the circle line is essentially back at punctual operation.

See the proceedings version for a comparison of the effects that holding and inserting a train from the same delayed direction in the next round are having on the passengers. It turns out that although the latter implies a voluntary partial cancellation of train trips, the travel times of the passengers increase less than when applying the holding response rule.

\section{Conclusions}

We presented a collection of response rules that are applicable in particular for circle metro lines after some relatively small disturbances occur. Moreover, we reported for twelve circle lines, which particular response rules are applied most often by the local experts. There, it turned out that holding and inserting a standby unit are selected rather frequently. In contrast, expressing and shortcuts are applied least often. Filling the gap can also be done by taking some train unit from another service - and then, of course, putting another train unit right back, in exchange. Even these advanced techniques are applied for more than half of the circle lines that we were investigating. For the research line of performing delay management with the help of mathematical optimization tools, we encourage the design 
teams of such future studies to include the application of more than just two or three of the response rules that we were presenting in this paper into their solution spaces.

Contrary to our initial intention, unfortunately, we are not able to report the operating performance of a sufficient number of circle lines. While the precise punctuality measures might differ from city to city, it could have been interesting to see, for which networks the punctuality of the circle line is superior, or inferior, compared to other lines in the very same network. Similarly, the intensity of regular reviews within the response team of past of its response action, cannot be reported for almost each circle line. Also the detailed framework within which the working times of the train drivers have to stay, had to stay beyond the scope of this survey, although we are considering it to be highly important, and sensible, at a time, especially for operations other than with GoA 4. It would have been interesting to check whether there would be any correlations between network features and intensity of team reviews on the one hand, versus the relative performance in terms of punctuality on the other hand - such an investigation has to stay up to future research.

Hence, we cannot present "the best" response rule, also because most of them require selected preconditions. There are certain network features that broaden the opportunities for the response team, e.g., having several crossovers in order to exchange two train units from the opposite directions in order to fill the gap, having additional sidings to let one train from the congestion wait for almost one round, or scheduling more train units (be it as dedicated standby units, or by increasing the time buffers during peak hours, as in Madrid). Recall that although scheduling one additional train unit decreases the efficiency, of course, together with the train units in continuous operation most likely the efficiency of this part of the fleet will not be lower than the one for a typical standard non-circle line.

Thus, in case that the punctuality of a circle line is significantly worse than the punctuality of the other lines in the same network, and provided that political decisionmakers are willing to support improvements by some additional funding, establishing some of these features would enrich the toolbox of the response team, and consequently could help to improve circle line's punctuality.

\section{Acknowledgements}

The authors would like to thank all the experts working for the networks that we were presenting in our study for spending their time in interviews with us. Moreover, we are thankful for the further support that we received from Jens Hebbe, Julie Jespersen-Groth, Hans-Christian Kaiser, Lingyun Meng, Lucia Mösch, Sukmun Oh, Jörn Pachl, Jonathan Tyler, Lingjiangfei $\mathrm{Wu}$, and Zixuan Zhao during the preparation and conduction of our study.

\section{References}

Bär, M., Dutsch, S., Fiebag, T., Gödde, M., Jin, S., Thieme, K. (2019): “Optimierung von Fahrgastwechselzeiten - Systematisierung von Maßnahmen zur Senkung der Fahrgastwechsel- und Haltezeiten in Stadtschnellbahnsystemen”. Der Nahverkehr, 12/2019, DVV Media Group, Hamburg, in German

Cadarso, L., Marín, 'A., Maróti, G. (2013): "Recovery of disruptions in rapid transit networks", Transportation Research Part E: Logistics and Transportation Review, 53, 15 -33 . 
Carrel, A., Mishalani, R.G., Wilson, N.H.M., Attanucci, J.P., Rahbee, A.B. (2010): "Decision Factors in Service Control on High-Frequency Metro Line: Importance in Service Delivery", Transportation Research Record, vol. 2146(1), pp. 52-59, https://doi.org/10.3141/2146-07

Chang, Y., Niu R., Wang, Y., Luan, X., Sama, M., d'Ariano, A. (2019): “Train Rescheduling for an Urban Rail Transit Line under Disruptions", In: Proceedings of the 8th International Conference on Railway Operations, Modelling and Analysis (ICROMA, RailNorrköping 2019), Editors: Peterson, A., Joborn, M., Bohlin, M., Linköping Electronic Conference Proceedings, ISBN 978-91-7929-992-7

Gao, Y., Kroon, L., Schmidt, M., Yang, L. (2016): "Rescheduling a metro line in an overcrowded situation after disruptions", Transportation Research Part B: Methodological, $93,425-449$.

Huang, Y., Mannino, C., Yang, L., Tang, T. (2020). "Coupling time-indexed and big-M formulations for real-time train scheduling during metro service disruptions", Transportation Research Part B: Methodological, 133, 38-61.

Kariyazaki, K., Hibino, N., Morichi, S. (2013). "Simulation Analysis of Train Operation to Recover Knock-On Delays under High-Frequency Intervals”, In: Proceedings of the 13th World Congress of Transport Research (WCTR 2013), Rio de Janeiro, Brazil

Kunimatsu, T., Hirai, C., Tomii, N., Takaba, M. (2009): "Evaluation of timetables by estimating passengers' personal disutility using micro-simulation”, RailZurich 2009, Zurich, Switzerland.

Lademann, F. (2001): "Bemessung von Begegnungsabschnitten auf eingleisigen S-BahnStrecken". PhD thesis, TU Darmstadt, Germany, in German

Li, S., De Schutter, B., Yang, L., Gao, Z. (2015): "Robust model predictive control for train regulation in underground railway transportation", IEEE Transactions on Control Systems Technology 24, 1075-1083.

Li, S., Dessouky, M.M., Yang, L., Gao, Z. (2017): "Joint optimal train regulation and passenger flow control strategy for high-frequency metro lines", Transportation Research Part B: Methodological 99, 113-137.

Puong, A.,Wilson, N.H.M. (2008): "A train holding model for urban rail transit systems", In: Computer-aided Systems in Public Transport, Editors: Hickman, M., Mirchandani, P., Voß, S., Springer LNEMS 600, pp. 319-337.

Shen, S., Wilson, N.H.M. (2001): "An Optimal Integrated Real-time Disruption Control Model for Rail Transit Systems", In: Proceedings of the 8th Conference on ComputerAided Scheduling of Public Transport (CASPT 2000), Editors: Voß, S., Daduna, J., Berlin, Germany, Springer LNEMS 505, ISBN 3-540-42243-9

Verkehrsverbund Berlin-Brandenburg (VBB, 2020): "S-Bahn Berlin - Teilnetze Nord-Süd und Stadtbahn (SBSNS-II), Verhandlungsverfahren mit Teilnahmewettbewerb, Anlage I, Anhang 1" (public tender), https://www.daisikomm.de/verfahren/D63399/ (Oct $\left.2^{\text {nd }}, 2020\right)$

Wang, Y., Zhang, M., Su, S., Tang, T., Ning, B., Chen, L. (2019): “An operation level based train regulation model for a metro line", In: 2019 IEEE Intelligent Transportation Systems Conference (ITSC), IEEE. pp. 2920-2925.

$\mathrm{Xu}, \mathrm{X} ., \mathrm{Li}, \mathrm{K}$. , Yang, L., (2016): "Rescheduling subway trains by a discrete event model considering service balance performance", Applied Mathematical Modelling 40, 1446 1466.

Yin, J., Wang, Y., Tang, T., Xun, J., Su, S. (2017): "Metro train rescheduling by adding backup trains under disrupted scenarios", Frontiers of Engineering Management, vol. 4(4), pp. 418-427, https: / /doi .org/10.15302/J-FEM-2017044 